\title{
Iniquidades socioeconômicas na conformação dos padrões alimentares de crianças e adolescentes ${ }^{1}$
}

\author{
Socioeconomic inequality shaping the dietary \\ patterns of children and teens
}

Rita de Cássia Ribeiro SILVA² Ana Marlúcia Oliveira ASSIS² Sophia Cornbluth SZARFARC ${ }^{3}$ Elizabete de Jesus PINTO ${ }^{4}$ Lília Carolina Carneiro da COSTA ${ }^{5}$ Laura Cunha RODRIGUES ${ }^{6}$

RES U M O

\section{Objetivo}

Identificar e quantificar a influência dos fatores socioeconômicos sobre os padrões alimentares.

\section{Métodos}

Estudo transversal de base populacional com amostra de 1136 crianças e adolescentes de 7 a 14 anos de idade, de ambos os sexos, matriculados na rede pública de Salvador (BA), Brasil. O consumo alimentar foi medido por meio do questionário qualitativo de frequência alimentar. Os padrões de consumo foram identificados por meio de análise de componentes principais. Para o estudo da influência dos indicadores socioeconômicos na conformação dos padrões alimentares, foram utilizados modelos de regressão quantílica.

\section{Resultados}

Os padrões alimentares extraídos foram classificados em padrão obesogênico e padrão tradicional. Nos modelos de regressão quantílica, ajustados por faixa etária e por sexo, o menor grau de instrução materna esteve

1 Apoio: Conselho Nacional de Desenvolvimento Científico e Tecnológico (Processo n 402462/2005-0) e Coordenação de Aperfeiçoamento de Pessoal de Nível Superior (Processo no 1254/10-3).

2 Universidade Federal da Bahia, Escola de Nutrição, Programa de Pós-Graduação em Alimentação e Nutrição, Departamento Ciência da Nutrição. Av. Araújo Pinho, 32, Canela, 40110-150, Salvador, BA, Brasil. Correspondência para/Correspondence to:

RCR SILVA. E-mail: <rcrsilva@ufba.br>.

3 Universidade de São Paulo, Faculdade de Saúde Pública. São Paulo, SP, Brasil.

${ }^{4}$ Universidade Federal da Bahia, Programa de Pós-Graduação da Medicina e Saúde. Salvador, BA, Brasil.

5 Universidade Federal da Bahia, Instituto de Matemática. Salvador, BA, Brasil.

${ }^{6}$ London School of Hygiene and Tropical Medicine, Department of Epidemiology and Population Health, London University. London, United Kingdon. 
452 RCR SILVA et al.

associado negativamente, em níveis significantes, na maioria dos percentis, ao consumo de alimentos que integram o padrão obesogênico. A baixa renda associou-se negativamente aos maiores percentis ( $p \geq 95)$. Os dados indicam não haver influência dos indicadores socioeconômicos sobre o consumo de alimentos que integram o padrão tradicional.

\section{Conclusão}

Conclui-se que há influência dos fatores socioeconômicos na adesão ao padrão obesogênico de consumo. Esse conjunto de resultados requer a atenção dos gestores públicos para a identificação de um padrão de consumo ocidental, visualizado amplamente nos estudos em que se avaliam padrões de consumo adotados na atualidade pela população brasileira - sobretudo por crianças e adolescentes -, caracterizados por englobar componentes alimentares de risco para as doenças crônicas não transmissíveis.

Termos de indexação: Adolescentes. Consumo de alimento. Crianças. Fatores socioeconômicas. Regressão quantílica.

\section{A B S T R A C T}

\section{Objective}

The present study identified and quantified the influence of socioeconomic factors on dietary patterns.

\section{Methods}

This cross-sectional, population-based study investigated the food habits of 1136 children and adolescents of both genders, aged 7 to 14 years, enrolled in public schools of Salvador (BA), Brazil, using the qualitative food frequency questionnaire. Intake patterns were identified by principal component analysis. Quantile regression models were used to study the influence of socioeconomic indicators on dietary patterns.

\section{Results}

The sample's eating patterns were classified into obesogenic or traditional. Quantile regression models adjusted for age and gender showed that, for most percentiles, low maternal education level was significantly negatively associated with consumption of foods included in the obesogenic pattern. Low income was negatively associated with the highest percentiles $(p \geq 95)$. The data showed that socioeconomic indicators do not influence the consumption of foods included in the traditional pattern.

\section{Conclusion}

Socioeconomic factors promote adherence to obesogenic eating patterns. These results indicate that public authorities need to identify the Western consumption pattern, commonly observed in studies that assess the current consumption patterns of the Brazilian population, especially of children and adolescents, characterized by foods that promote non-communicable chronic diseases.

Indexing terms: Adolescent. Consumption. Child. Socioeconomic factors. Quantile regression

\section{N T R O D U ÇÃ O}

Substanciais mudanças vêm ocorrendo no perfil de saúde da população brasileira. Análise preliminar das tendências de indicadores dos níveis globais de saúde indica o crescimento das prevalências das doenças crônicas não degenerativas, o que representa grande desafio para os sistemas de saúde de todo o mundo. Embora informações sobre a ocorrência desses eventos entre crianças e adolescentes sejam escassas no Brasil, é possível supor, pelo aumento da prevalência de sobrepeso e de obesidade nessa etapa da vida, que as estatísticas se aproximam daquelas registradas para a população adulta'. Vários fatores têm sido associados à ocorrência desses eventos; dentre eles, destacam-se as mudanças associadas ao estilo de vida, em que se incluem padrões alimentares inadequados associados à inatividade física, uso abusivo do álcool e tabagismo². No que diz respeito às mudanças dos padrões alimentares, as alterações de maior destaque referem-se ao aumento da oferta de alimentos industrializados com alta densidade energética, à custa de gor- 
duras saturadas e carboidratos simples, em detrimento daqueles de origem vegetal, que apresentam baixo valor calórico, são fontes de micronutrientes e fibras ${ }^{3}$. A escolha alimentar é um processo complexo, influenciado tanto por fatores biológicos quanto por fatores sociais, culturais e econômicos ${ }^{4}$. As iniquidades socioeconômicas parecem interferir na conformação dos padrões alimentares do brasileiro. Poucos são os estudos que buscam identificar e quantificar a influência dos fatores socioeconômicos sobre os padrões alimentares, sobretudo entre crianças e adolescentes. Ademais, os resultados disponíveis não são consensuais ${ }^{5-8}$. Tendo em vista essa lacuna no conhecimento, explorar possíveis relações entre consumo alimentar e situação socioeconômica poderá contribuir para a compreensão da tendência crescente da prevalência de excesso de peso e de obesidade nesse ciclo da vida. Além disso, estudos nessa área poderão subsidiar a formulação e a implementação de estratégias voltadas para a promoção de alimentação saudável e da saúde.

\section{MÉ T O D O S}

Trata-se de um estudo transversal do qual participaram 1136 estudantes de 7 a 14 anos, de ambos os sexos. Esses estudantes foram identificados em uma investigação mais ampla, que teve por objetivo estudar fatores associados à anemia ferropriva em crianças e adolescentes matriculados na rede pública de ensino da cidade de Salvador (BA) ${ }^{9}$. O processo de amostragem no estudo original envolveu desenho complexo, valendo-se da estratificação das escolas em dois níveis (estadual e municipal), seguida pelo procedimento de amostragem por conglomerado em três estágios, conforme descrito a seguir: o primeiro estágio representado pelos distritos sanitários; o segundo, pelas escolas; o terceiro, pelos alunos.

Em virtude de questões logísticas de campo, as informações dos estudantes selecionados foram extraídas de seis dos 12 distritos existentes em Salvador, cidade em que se observaram 117 escolas estaduais e 173 municipais. As escolas estaduais comportavam 58059 alunos, e as municipais, 56 555. Para atender ao número amostral previamente definido, verificou-se a necessidade de selecionar dez alunos de cada uma das 58 escolas municipais e 23 alunos de cada uma das 27 escolas estaduais. Os alunos foram ordenados por série $\left(1^{\mathrm{a}}\right.$ a $\left.8^{\mathrm{a}}\right)$ para posterior amostragem sistemática para a seleção dos 1200 estudantes.

\section{Padrão de consumo alimentar}

O Questionário Quantitativo de Frequência Alimentar (QQFA), elaborado por Slater et al. ${ }^{10}$, foi adaptado e adotado para a avaliação da frequência de consumo alimentar dos estudantes. Trata-se de um instrumento validado por Voci et al. ${ }^{11}$. Constam do QQFA 132 itens alimentares, tendo sido adicionados alimentos regionais. Um álbum com desenhos de porções diferenciadas dos alimentos e utensílios foi utilizado na coleta da informação de consumo. A frequência de consumo desses itens foi dividida em quatro categorias, assim codificadas: nunca consome $=0 ; 1$ a 3 vezes ao mês $=1 ; 1$ a 4 vezes na semana $=2$; 5 a 7 vezes na semana $=3$. Em seguida, foram somadas as frequências codificadas correspondentes aos alimentos efetivamente consumidos pelo indivíduo em cada grupo alimentar, o que constituiu o numerador das medidas-resumo. $\mathrm{O}$ denominador correspondeu ao número máximo de alimentos que o indivíduo poderia consumir em cada grupo de alimentos multiplicado por $4^{12}$.

Os alimentos ou grupos de alimentos que tiveram consumo acima de 15\% integraram a análise fatorial e foram agrupados em 17 categorias conforme a semelhança de conteúdo nutricional: leite e derivados: leite in natura, leite em pó, iogurte, queijo; carnes e derivados: carne de boi, carne de sertão, frango, fígado, miúdo, peixe, embutido, fato; ovos: ovo cozido, ovo frito, omelete; cereais e derivados: arroz, biscoito doce, biscoito salgado, biscoito cream cracker, bolo, cuscuz 
de milho, farinha de mandioca, macarrão, pão, pipoca salgada, pipoca doce; leguminosas: amendoim, feijão; verduras: alface, abóbora, chuchu, cenoura, quiabo, pepino, repolho, sopa de verdura; raízes e tubérculos: aipim, batata-doce, batata; frutas: abacaxi, abacate, acerola, banana da terra, banana prata, coco, laranja, limão, maçã, mamão, manga, maracujá, melancia, uva, tangerina, jaca, suco de frutas; óleo e gordura: manteiga, margarina, leite de coco, bacon; açúcares e doces: açúcar, chocolate, goiabada, sorvete, geladinho; frituras e sanduíches: frituras salgadas (coxinha, pastel etc.), batata frita, hambúrguer, misto, cachorro-quente, pizza, salgadinho; preparações típicas: abará, acarajé, caruru, feijão tropeiro, feijoada, moqueca; bebidas: refrigerantes, café, água de coco, achocolatado, suco artificial.

\section{Características das condições socioeconômicas e demográficas}

A condição socioeconômica da família foi avaliada com base na renda familiar mensal e na escolaridade materna. A renda foi traduzida em salário-mínimo vigente no período do estudo $(R \$ 380,00)$, sendo categorizada em dois estratos: $<1$ salário-mínimo e $\geq 1$ salário-mínimo (categoria de referência) A escolaridade materna foi categorizada em dois níveis, conforme a última série escolar cursada: I $<5^{\text {a }}$ série, $\| \geq 5^{\text {a }}$ série $_{\text {(categoria de referência) }}$. As variáveis demográficas foram: sexo (masculino $_{\text {(categoria de referenncia)' }}$ feminino) e faixa etária $(<10$ $\operatorname{anos}_{\text {(categoria de referência) }} \mathrm{e} \geq 10$ anos).

\section{Aspectos éticos}

Parecer favorável quanto à pertinência ética do estudo foi concedido pelo Comitê de Ética do Instituto de Saúde Coletiva da Universidade Federal da Bahia, sob registro 006- 06/CEP-ISC. Após o conhecimento dos objetivos do estudo, os pais ou responsáveis pelo escolar que concordaram com a participação do filho na pesquisa assinaram o Termo de Consentimento Livre e Esclarecido; os analfabetos concederam sua autorização por meio da impressão digital.

\section{Análise estatística}

Antes de proceder à análise fatorial exploratória, estimou-se o coeficiente Kaiser-Mayer-Olkin (KMO) e aplicou-se o teste de esfericidade de Bartlett para indicar a adequação dos dados à análise ${ }^{12}$. Realizou-se a análise de componentes principais, seguida da rotação ortogonal (varimax) para examinar a estrutura (padrão) fatorial exploratória. O número de fatores extraídos foi guiado pelas recomendações do Hearty \& Gibney ${ }^{13}$. A consistência interna das dimensões do QQFA foi avaliada, sendo considerado aceitável índice alpha de Cronbach $>0,65$. Modelou-se a regressão quantílica a fim de avaliar os determinantes socioeconômicos na conformação dos padrões alimentares. Os escores gerados na análise fatorial integraram o modelo na forma contínua.

Modelos de regressão quantílica foram construídos para identificar a associação entre os padrões alimentares e as covariáveis (renda, escolaridade, sexo e idade). A técnica semiparamétrica de regressões quantílicas tem despertado a atenção de numerosos pesquisadores, sendo usa-da como instrumental em diversas constatações empíricas. A técnica de regressão quantílica desenvolvida por Koenker \& Basset $^{14}$, além de não necessitar da suposição básica de mínimos quadrados, é mais informativa, pois permite estimar toda a distribuição condicional da variável dependente, por meio dos quantis da distribuição, podendo-se obter a regressão para cada quantil de interesse. Portanto, a regressão quantílica propicia um estimador mais robusto quando a distribuição dos erros é heterocedástica e/ou não gaussiana. Por fim, os modelos de regressão quantílica são aditivos, ou seja, os coeficientes de regressão são interpretados como a mudança num dado percentil da variável dependente provocado pela mudança de uma unidade da variável independente. 
No presente estudo, a escolha do modelo justifica-se pela assimetria à direita da curva de ingestão dos alimentos que integram os padrões alimentares estudados (variáveis dependentes) e pela heterocedasticidade dessas variáveis em relação às variáveis explicativas. Para avaliar a associação dos indicadores socioeconômicos com os padrões alimentares, foram construídos dois modelos de regressão: um para cada padrão identificado. Foram estimados, simultaneamente, coeficientes de regressão a cada intervalo dos percentis a partir do mesmo modelo para analisar o efeito das variáveis independentes ao longo de toda a distribuição. O erro-padrão para cada um dos coeficientes estimados foi computado a partir de 1000 replicações por meio da técnica de replicação bootstrap. Foram consideradas, no modelo, como variáveis associadas aquelas cujo Intervalo de Confiança (IC) de 95\% não continha o zero. O pacote estatístico Stata (Statistics/Data Analysis, versão 10.1) foi usado para análise.

\section{RES U LTA D O S}

Do total inicial de 1205 , registrou-se a perda de 69 indivíduos (5,3\%), resultando em uma amostra de 1136 estudantes. A perda deve-se à recusa em participar, à mudança da criança para outra cidade ou à transferência para outra escola. Das 1136 crianças e adolescentes investigados,
$49,2 \%$ são do sexo feminino e a maioria $(73,0 \%)$ tinha de 7 a 9 anos de idade. A renda mensal menor do que um salário-mínimo/mês foi identificada em 23,9\% das famílias. Quanto ao nível de escolaridade, $28,4 \%$ das mães tinham o ensino fundamental (incompleto ou completo) (dados não apresentados).

O índice de confiança da análise fatorial foi verificado por meio do coeficiente $\mathrm{KMO}(0,873)$ e do teste de esfericidade de Bartlett (3 243,17; $p<0,001)$. Esses valores indicaram que as correlações entre os itens eram suficientes e adequadas para o processamento da análise fatorial. Dessa análise, foram extraídos dois padrões que representaram $44,5 \%$ da variância total. O número de fatores extraídos foi definido por critérios estatísticos (screeplot e autovalores $\geq 1,3$ ) e pelo significado conceitual dos padrões identificados. Os padrões alimentares extraídos foram classificados como: padrão obesogênico e padrão tradicional (Tabela 1). A escala geral e as subescalas do alpha de Cronbach para avaliar a consistência interna dos dados foram acima de $0,65(\alpha>0,65)$, garantindo, assim, o nível aceitável de precisão de medida para os dois padrões, e assegurando a consistência interna para as dimensões do questionário utilizado. O grupo das leguminosas não foi considerado nas análises devido à baixa comunalidade $\left(h^{2}\right)$ apresentada.

Tabela 1. Distribuição das cargas fatoriais dos padrões de consumo alimentar. Salvador (BA), 2008.

\begin{tabular}{lccc}
\hline Alimentos e grupos de alimentos & Padrão obesogênico & Padrão tradicional & h2 \\
\hline Carnes e derivados & - & 0,577 & 0,382 \\
Ovos & - & 0,492 & 0,262 \\
Cereais e derivados & - & 0,538 & 0,469 \\
Verduras & - & 0,723 & 0,590 \\
Raízes e tubérculos & - & 0,711 & 0,548 \\
Frutas & - & 0,577 & 0,584 \\
Leites e derivados & 0,507 & - & 0,292 \\
Óleos e gorduras & 0,441 & - & 0,217 \\
Açúcares e doces & 0,768 & - & 0,599 \\
Frituras e sanduíches & 0,679 & - & 0,478 \\
Preparações típicas & 0,655 & - & 0,463 \\
Bebidas & 0,638 & - & 0,441 \\
\% Variância explicada & 34,61 & 9,890 & 44,50 \\
\hline
\end{tabular}

$h^{2}$ : baixa comunalidade. 
As Figuras 1 , 2, 3, 4 mostram as mudanças nos coeficientes de regressão ao longo da distribuição em relação à renda familiar e à escolaridade materna, ajustados por sexo e por faixa etária. Esses coeficientes expressam a diferença de consumo (em escores) entre as categorias das variáveis independentes analisadas. Nesses gráficos, a área cinza representa o IC de 95\% dos coeficientes estimados em cada percentil. Nos modelos de regressão quantílica, ajustados pela faixa etária e pelo sexo, o menor grau de escolarização materna esteve associado negativamente ao padrão obesogênico, em níveis significantes, na maioria dos percentis. A baixa renda associouse negati-vamente aos maiores percentis $(p \geq 95)$ (Figuras 1 e 2). Os dados apontam não haver associação entre grau de instrução materna e consumo de alimentos que integram o padrão tradicional. Porém, a baixa renda associou-se negativamente, com significância boderline, aos menores percentis ( $p \leq 25$ ) (Figuras 3 e 4).

\section{I S CUSS Ã O}

O presente estudo permitiu caracterizar dois padrões de consumo alimentar entre as crianças e os adolescentes da cidade de Salvador (BA): tradicional e obesogênico. O padrão obesogênico, desfavorável ao crescimento saudável de crianças e adolescentes, sobretudo por integrar alimentos-fonte de gorduras em geral e de gorduras saturadas, assim como alimentos de alto índice glicêmico, similarmente registrado por outros estudos $3,5,15,16$, pode responder, em parte, pelos fatores de risco associados às doenças crônicas não transmissíveis nesse ciclo de vida ${ }^{17-19}$.

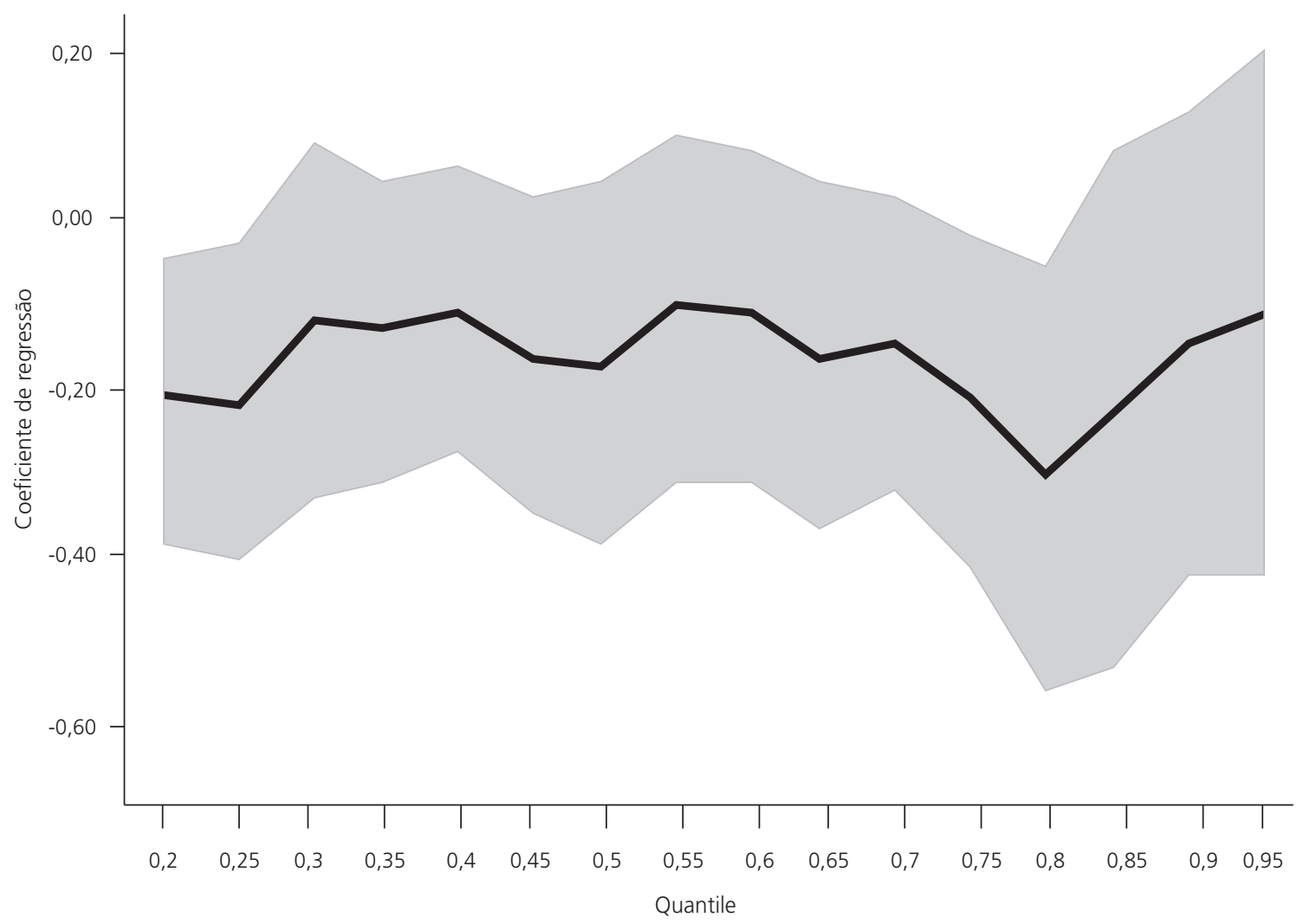

Figura 1. Coeficientes de regressão quantílica para avaliação da associação entre escolaridade materna e padrão de consumo obesogênico. Salvador (BA), 2008. 


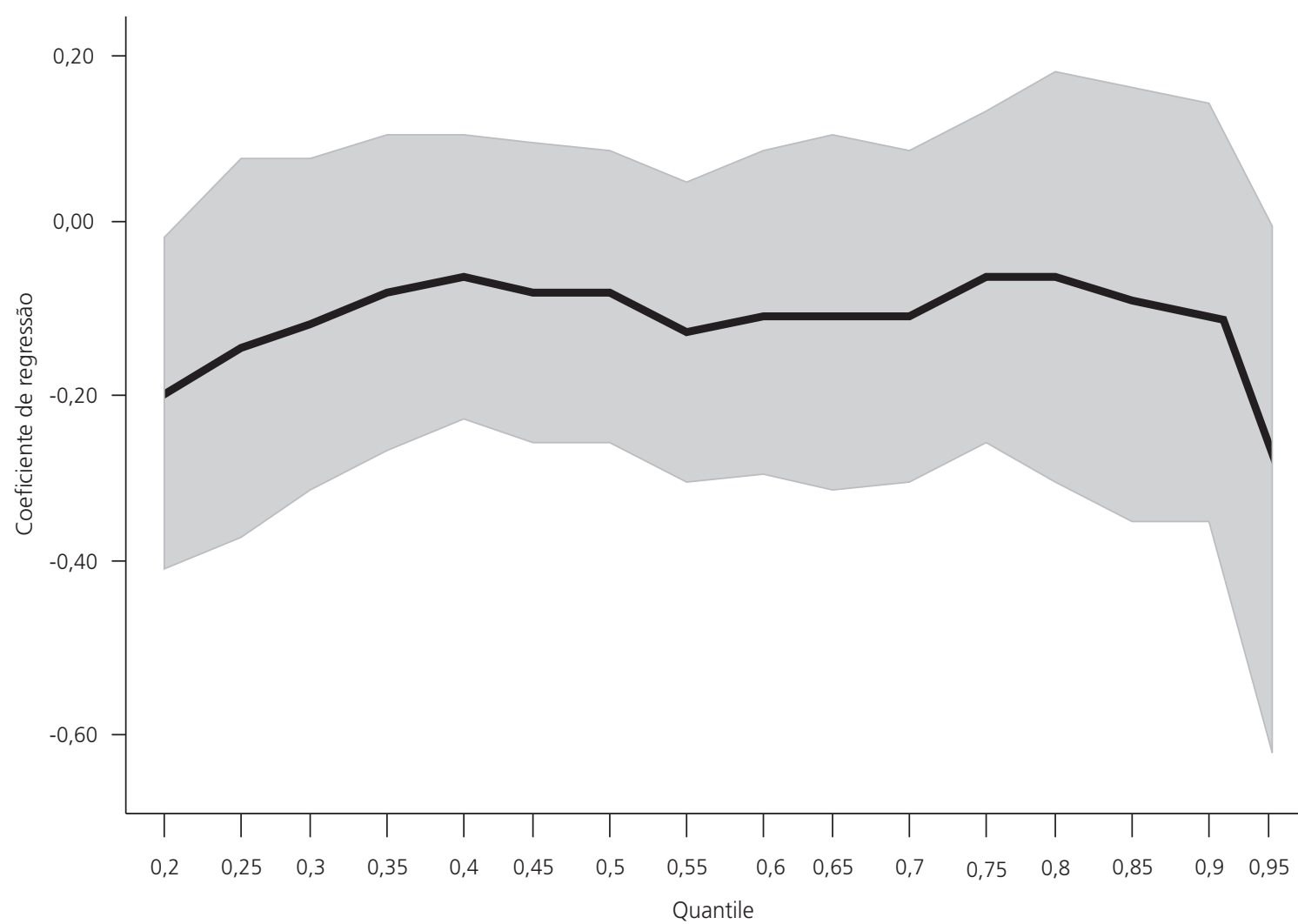

Figura 2. Coeficientes de regressão quantílica para avaliação da associação entre renda familiar e padrão de consumo obesogênico. Salvador (BA), 2008.

No presente estudo, as análises multivariadas mostram a influência da instrução materna e da renda familiar sobre a adesão ao padrão obesogênico de consumo alimentar; o menor grau de escolaridade materna esteve negativamente associado, de maneira significante, em todos os percentis, a esse padrão, enquanto a menor renda se associou negativamente aos percentis finais da distribuição ( $p \geq 95$ ). A escassez de estudos que utilizam a mesma metodologia de avaliação de consumo e com enfoque na mesma faixa etária torna mais difícil a comparação dos achados. Corroboram os resultados aqui apresentados os daqueles estudos que indicam que, à medida que o nível socioeconômico melhora, as escolhas voltam-se para dietas com altas concentrações energéticas, compostas de alimentos-fonte de gorduras saturadas e açúcares simples ${ }^{5,20,21}$. Contudo, esses resultados não são consensuais. Xie et al. ${ }^{6}$ e Aranceta et al. ${ }^{7}$ verificaram que o consumo de dietas com altos teores de gordura é maior entre jovens de mais baixo nível socioeconômico.

Relata-se que a associação da melhor situação socioeconômica com o consumo de alimentos que integram o padrão obesogênico seja mediada pelas mudanças - impostas, sobretudo, pelo estilo de vida moderno - vivenciadas nas últimas décadas pelas famílias brasileiras. No mundo agitado de trabalho em que vivem atualmente as famílias, os pais dedicam pouco tempo aos filhos, e menos ainda a sua alimentação, e recompensam sua ausência com passeios a restaurantes do tipo fast-food ou com a compra de guloseimas e refrigerantes, que, em sua maioria, são pouco saudáveis ${ }^{22}$.

Há ainda a considerar que a mulher passou a se dividir entre o trabalho fora de casa e as 


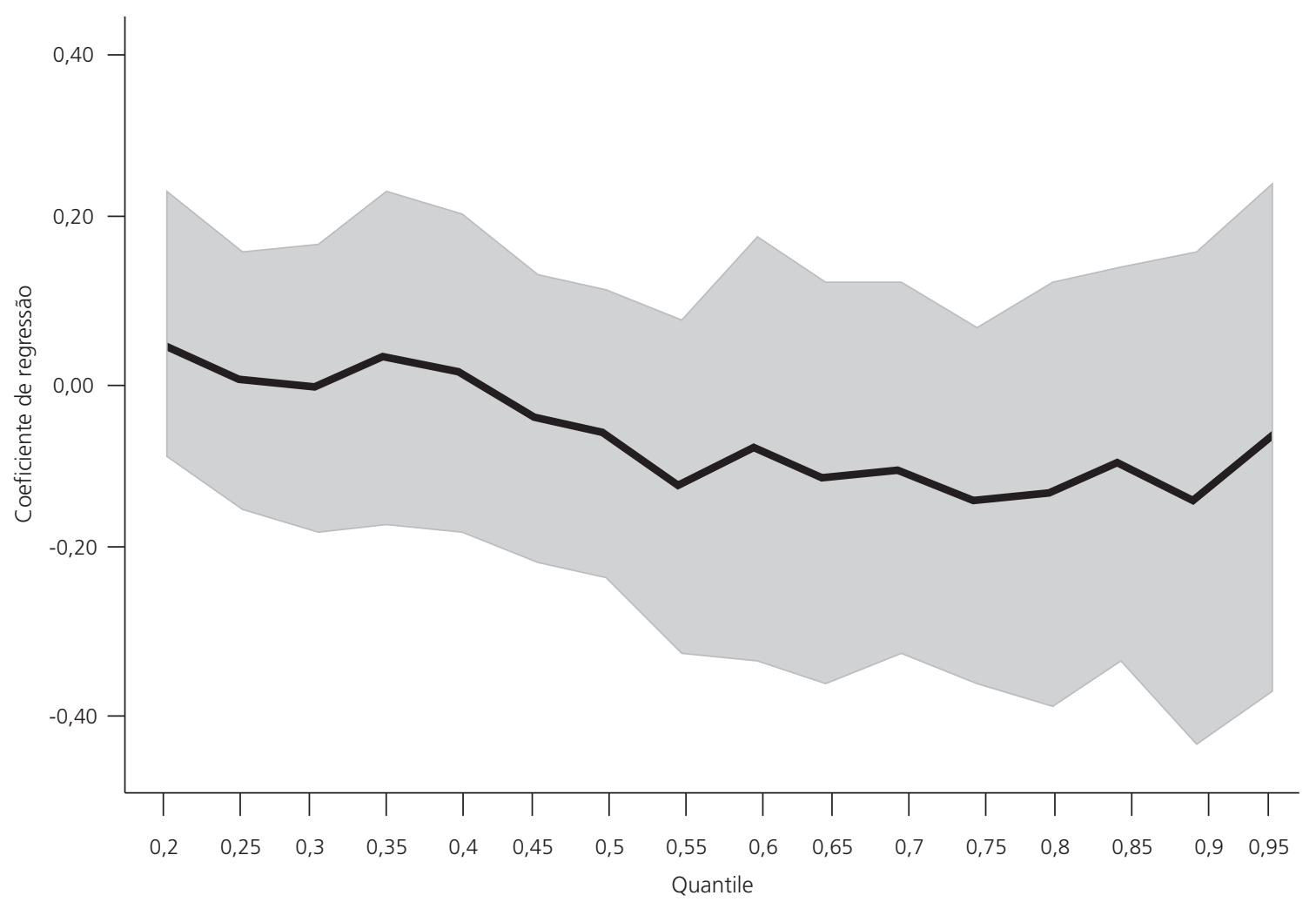

Figura 3. Coeficientes de regressão quantílica para avaliação da associação entre escolaridade materna e padrão de consumo tradicional. Salvador (BA), 2008.

tarefas domésticas, e necessita de alimentos práticos e de rápido preparo para sua família, muitos deles produtos industrializados, que se tornaram de fácil acesso 22 . Para alguns autores, a alimentação fora de casa, o crescimento da oferta de refeições rápidas (fast food) e a ampliação do uso de alimentos industrializados/processados estão vinculados diretamente à renda das famílias e às possibilidades de gasto com alimentação em particular, e estão associados ao valor sociocultural que os alimentos apresentam para cada grupo social4,22.

Os indicadores socioeconômicos estudados não se associou ao consumo alimentar caracterizado por um padrão tradicional e mais saudável. Esse resultado é compatível com aquele observado para o Brasil, em que se identificou declínio no consumo de alimentos básicos, em especial o arroz e feijão ${ }^{23}$, em todas as classes econômicas. Esses resultados levam a supor fragilidade de cobertura dos programas de atenção básica em promover ações de promoção de alimentação saudável com orientações de uma alimentação diversificada, com resgate de uma alimentação à base da combinação entre feijão e arroz. Não é possível ainda descartar que outros fatores não mencionados neste estudo - e que podem ser objeto de outras investigações - estejam determinando a negativa adesão a tal padrão nesse ciclo da vida.

É importante destacar as limitações deste estudo e o fato de ele ser do tipo transversal, em que não é possível estabelecer relações causais por não se contemplar a sequência temporal entre a exposição e o efeito. Entretanto, os resultados encontrados são compatíveis com os registros da 


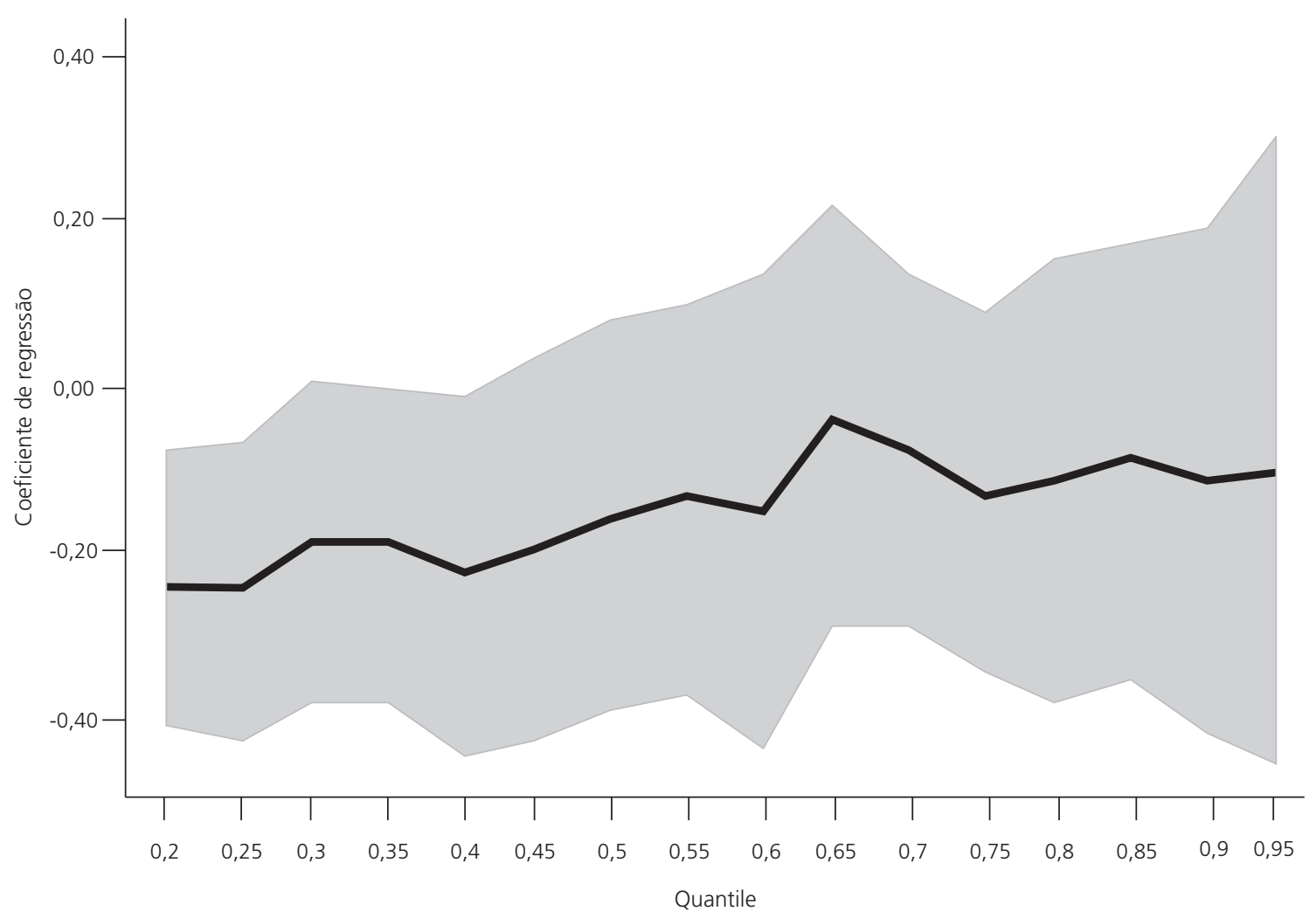

Figura 4. Coeficientes de regressão quantílica para avaliação da associação entre renda familiar e padrão de consumo tradicional. Salvador (BA), 2008.

literatura consultada em que muitos autores concluem pela iniquidade como fator de adesão aos diferentes padrões alimentares 5,20,21. Também o uso arbitrário de extração do número de fatores retidos e o método de rotação da matriz de correlação da análise fatorial podem ser outra limitação do estudo. Contudo, essas decisões foram tomadas levando-se em consideração os objetivos da pesquisa e a interpretabilidade dos dados, conforme recomendado por Hearty \& Gibney ${ }^{13}$.

Vale ressaltar, ainda, que o caráter probabilístico e a implementação bem-sucedida dos procedimentos empregados para a seleção amostral apontam que os resultados do presente estudo podem ser estendidos para o conjunto de crianças e adolescentes, de 7 a 14 anos de idade, matriculados na rede pública de ensino do município de Salvador.
C O N C L U S Ã O

Independentemente das limitações consideradas, entende-se que os resultados do presente estudo favorecem a compreensão e a explicação da relação entre condições socioeconômicas e padrões alimentares. Conclui-se pela influência dos fatores socioeconômicos na adesão ao padrão obesogênico de consumo por crianças e adolescentes. Esse conjunto de resultados deve requerer a atenção dos gestores públicos para a identificação de um padrão de consumo ocidental, visualizado amplamente nos estudos em que se avaliam padrões de consumo adotados na atualidade pela população brasileira em geral, caracterizados pela ingestão de cereais refinados, bebidas com adição de açúcar, frituras, que são considerados como componentes alimentares de risco para as doenças crônicas não transmissíveis. 
Assim constatado, é imperativo o monitoramento das práticas alimentares voltadas para a promoção da alimentação saudável da população, em especial nessa etapa da vida. Diante da vulnerabilidade dos escolares para a adoção de hábitos alimentares inadequados, a escola se configura como um ambiente privilegiado para a promoção da saúde dos jovens.

\section{COLABORADORES}

RCR SILVA participou da concepção do estudo, análise dos dados, interpretação dos resultados e redação do manuscrito. AMO ASSIS participou da interpretação dos resultados e redação do manuscrito. SC SZARFARC fez a revisão do manuscrito. EJ PINTO fez a limpeza e sistematização do banco para análise dos dados. L COSTA fez análise dos dados. LC RODRIGUES participou da interpretação dos resultados e revisão do manuscrito.

\section{REFERÊ NCIAS}

1. Brazilian Institute of Geography and Statistics. POF 2008-2009: anthropometry and nutritional status of children, adolescents and adults in Brazil [Internet]. Rio de Janeiro: Brazilian Institute of Geography and Statistics; 2010 [cited 2011 Mar 5]. Available from <http://www.ibge.gov.br>.

2. Holt R. The Food and Agriculture Organization/ World Health Organization expert report on diet, nutrition and prevention of chronic diseases. Diabetes Obes Metab. 2003; 5(5):354-8.

3. Carmo MB, Toral N, Silva MV, Slater B. Consumo de doces, refrigerantes e bebidas com adição de açúcar entre adolescentes da rede pública de ensino de Piracicaba, São Paulo. Rev Bra Epidemiol. 2006; 9(1):121-30.

4. Lenz A, Olinto MTA, Dias-da-Costa JS, Alves AL, Balbinotti M, Pattussi MP, et al. Socioeconomic, demographic and lifestyle factors associated with dietary patterns of women living in Southern Brazil. Cad Saúde Pública. 2009; 25(6):1297-306.

5. Neutzling MB, Araújo CLP, Vieira MFA, Hallal PC, Menezes AM. Frequencia de consumo de dietas ricas em gordura e pobres em fibras entre adolescentes. Rev Saúde Pública. 2007; 41(3):336-42.
6. Xie B, Gilliland FD, Li YF, Rockett HR. Effects of ethnicity, family income, and education on dietary intake among adolescents. Prev Med. 2003; 36(1): 30-40.

7. Aranceta J, Perez-Rodrigo C, Ribas L, Serra-Majem L. Sociodemographic and lifestyle determinants of food patterns in Spanish children and adolescents: the enKid study. Eur J Clin Nutr. 2003; 57(Suppl 1): s40-4.

8. Levy RB, Castro IRR, Cardoso LD, Tavares LF, Sardinha LMV, Gomes FD, et al. Food consumption and eating behavior among brazilian adolescents: National Adolescent School-based Health Survey (PeNSE), 2009. Ciênc Saúde Coletiva. 2010; 15 (Suppl 2):3085-97.

9. Borges CQ, Silva RCR, Assis AMO, Pinto EJ, Fiaccone $\mathrm{RL}$, Pinheiro SMC. Fatores associados à anemia em crianças e adolescentes de escolas públicas de Salvador (BA), Brasil. Cad Saúde Pública. 2009; 25(4):877-88.

10. Slater B, Philippi ST, Fisberg RM, Latorre MR. Validation of a semi-quantitative adolescent food frequency questionnaire applied at a public school in São Paulo, Brazil. Eur J Clin Nutr. 2003; 57(5): 629-35.

11. Voci SM, Slater B, Silva MV, Marchioni DM, Latorre MD. Estudo de calibração do Questionário de Frequência Alimentar para Adolescentes (QFAA). Ciênc Saúde Coletiva. 2011;16(4):2335-43.

12. Neumann AICP, Martins IS, Marcopito LF, Araújo EAC. Padrões alimentares associados a fatores de risco para doenças cardiovasculares entre residentes de um município brasileiro. Rev Panam Salud Pública. 2007; 22(5):329-39.

13. Hearty AP, Gibney MJ. Comparison of cluster and principal component analysis techniques to derive dietary patterns in Irish adults. Br J Nutr. 2009; 101(4):598-608.

14. Koenker R, Bassett G. Regression quantiles. Econometrica.1978; 46:33-50.

15. Silva ARV, Damasceno MMC, Marinho NBP, Almeida LS, Araújo MFM, Almeida PC, et al. Hábitos alimentares de adolescentes de escolas públicas de Fortaleza, CE, Brasil. Rev Bras Enferm. 2009; 62:18-24.

16. Leal GV, Philippi ST, Matsudo SM, Toassa EC. Consumo alimentar e padrão de refeições de adolescentes, São Paulo, Brasil. Rev Bras Epidemiol. 2010; 13(3):457-67.

17. Farias Júnior JC, Nahas MV, Barros MVG, Loch MR, Oliveira ESA, De Bem MFL, et al. Comportamento de risco à saúde em adolescentes no sul do Brasil: 
prevalência e fatores associados. Rev Panam Salud Publica. 2009; 25(4):344-52.

18. Mikkila V, Rasanen L, Raitakari OT, Pietinen P, Viikari J. Consistent dietary patterns identified from childhood to adulthood: the cardiovascular risk in Young Finns Study. Br J Nutr. 2005; 93(6):923-31.

19. Pinto SL, Silva RC, Priore SE, Assis AM, Pinto EJ. Prevalência de pré-hipertensão e de hipertensão arterial e avaliação de fatores associados em crianças e adolescentes de escolas públicas de Salvador, Bahia, Brasil. Cad Saúde Pública. 2011; 27(6): 1065-75.

20. Nunes MMA, Figueiroa JN, Alves JGB. Excesso de peso, atividade física e hábitos alimentares entre adolescentes de diferentes classes econômicas em Campina Grande (PB). Rev Assoc Med Bras. 2007; 53(2):130-4.
21. Sarlio-Lahteenkorva S, Lahelma E. The association of body mass index with social and economic disadvantage in women and men. Int J Epidemiol. 1999; 28(3):445-9.

22. Mendonça CP, Anjos LA. Aspectos das práticas alimentares e da atividade física como determinantes do crescimento do sobrepeso/obesidade no Brasil. Cad Saúde Pública. 2004; 20:698-709.

23. Levy-Costa RB, Sichieri R, Pontes NS, Monteiro CA. Disponibilidade domiciliar de alimentos no Brasil: distribuição e evolução (1974-2003). Rev Saúde Pública. 2005; 39(4):530-40.

Recebido em: 19/10/2011

Versão final em: 25/5/2012

Aprovado em: 6/6/2012 
